\title{
Causal judgment from contingency information: A systematic test of the $p$ CI rule
}

\author{
PETER A. WHITE \\ Cardiff University, Cardiff, Wales
}

\begin{abstract}
Contingency information is information about the occurrence or nonoccurrence of an effect when a possible cause is present or absent. Under the evidential evaluation model, instances of contingency information are transformed into evidence and causal judgment is based on the proportion of relevant instances evaluated as confirmatory for the candidate cause. In this article, two experiments are reported that were designed to test systematic manipulations of the proportion of confirming instances in relation to other variables: the proportion of instances on which the candidate cause is present, the proportion of instances in which the effect occurs when the cause is present, and the objective contingency. Results showed that both unweighted and weighted versions of the proportion-of-confirmatoryinstances rule successfully predicted the main features of the results, with the weighted version proving more successful. Other models, including the power PC theory, failed to predict the results.
\end{abstract}

Suppose you eat a series of meals with various ingredients. Sometimes you suffer from indigestion and sometimes you do not. After a while, you notice that indigestion is likely to occur if the meal included broccoli and is not likely to occur if there was no broccoli in the meal. Eventually, you conclude that broccoli causes you to have indigestion. This example illustrates the activity of causal induction from contingency information. You begin with no causal belief, but you acquire one from a series of observations of things that go together.

Contingency information concerns effects that occur or do not occur and possible causes that may be present or absent. This kind of information can be represented in a $2 \times 2$ table, such as Table 1 . It is generally accepted that the objective contingency for information in this form is described by the $\Delta P$ rule, shown in Equation 1 (Jenkins \& Ward, 1965; McKenzie, 1994; Ward \& Jenkins, 1965). In Equation 1, $\Delta P$ is defined in terms of the conventional letters shown in Table 1 . Values of $\Delta P$ range from -1 (perfect negative contingency) to +1 (perfect positive contingency).

$$
\Delta P=a /(a+b)-c /(c+d)
$$

Currently, there are three general classes of models of causal judgment. In one class, instances of contingency information contribute to the development of an associative bond between a cause and an effect and causal judgment is based on the strength of the associative bond. This is exemplified by the application of the Rescorla-Wagner

I am grateful to Mike Oaksford and two anonymous reviewers for helpful comments on an earlier draft of this paper. Correspondence concerning this article should be addressed to P. A. White, School of Psychology, Cardiff University, P.O. Box 901, Cardiff CF103YG, Wales (e-mail: whitepa@cardiff.ac.uk). model to human causal judgment (Allan, 1993; Rescorla \& Wagner, 1972; Shanks, 1993, 1995; Shanks \& Dickinson, 1987; Wasserman, Kao, Van Hamme, Katagiri, \& Young, 1996). In a second class, causal judgments are generated by an inductive rule operating on a body of contingency information. This is exemplified by the proposal that the $\Delta P$ rule itself is the inductive rule of causal judgment (Cheng \& Novick, 1990, 1992) and by the power PC theory (Cheng, 1997). The present research is concerned primarily with the third kind of model: the evidential evaluation (EE) model (White, 2000b, 2002a, 2002b, 2002c, 2003a, 2003b, 2003c).

Under the EE model, instances of contingency information are initially interpreted as evidence - that is, they are assessed as having a certain degree of confirmatory or disconfirmatory value for the proposition that the cause produces the effect. Causal judgment is derived from the overall confirmatory or disconfirmatory weight of the evidence. Asymptotic judgments - that is, final judgments after a series of instances has been presented - are modeled by Equation 2. These are the concern of the present research.

$$
p C I=\frac{\mathrm{w}_{a}\left[\mathrm{~s}\left(\mathrm{x}_{a}\right)\right]+\mathrm{w}_{d}\left[\mathrm{~s}\left(\mathrm{x}_{d}\right)\right]+\mathrm{w}_{b}\left[\mathrm{~s}\left(\mathrm{x}_{b}\right)\right]+\mathrm{w}_{c}\left[\mathrm{~s}\left(\mathrm{x}_{c}\right)\right]}{a+b+c+d}
$$

In Equation 2, $p$ CI stands for the proportion of confirmatory instances. $\mathrm{s}\left(\mathrm{x}_{a}\right), \mathrm{s}\left(\mathrm{x}_{b}\right), \mathrm{s}\left(\mathrm{x}_{c}\right)$, and $\mathrm{s}\left(\mathrm{x}_{d}\right)$ are subjective evaluations of cells $a, b, c$, and $d$, respectively, and vary from -1 (disconfirmatory extreme) to +1 (confirmatory extreme). $\mathrm{w}_{a}, \mathrm{w}_{b}, \mathrm{w}_{c}$, and $\mathrm{w}_{d}$ are weights assigned to $\mathrm{s}\left(\mathrm{x}_{a}\right)$, $\mathrm{s}\left(\mathrm{x}_{b}\right), \mathrm{s}\left(\mathrm{x}_{c}\right)$, and $\mathrm{s}\left(\mathrm{x}_{d}\right)$, respectively, and vary from 0 to $1 .{ }^{1}$

That is the general form of the model designed to accommodate individual differences in notions of evidential value. Normatively, cells $a$ and $d$ are confirmatory 
Table 1

Conventional Identification of Cells in the $2 \times 2$ Contingency Table

\begin{tabular}{ccc}
\hline & \multicolumn{2}{c}{ Effect Occurs: } \\
\cline { 2 - 3 } Candidate Cause & Yes & No \\
\hline Present & $a$ & $b$ \\
Absent & $c$ & $d$ \\
\hline
\end{tabular}

and cells $b$ and $c$ are disconfirmatory. Notions of evidential value vary between individuals but, in the aggregate, show a reasonable degree of conformity to these normative notions (White, 2000a). In previous studies, the unweighted version of the $p$ CI rule with values of +1 for cells $a$ and $d$ and -1 for cells $b$ and $c$ has been tested, and strong support for it was found (White, 2000b, 2002a, 2002b, 2002c, 2003a, 2003b, 2003c).

Although this model bears a superficial resemblance to linear combination models such as Schustack and Sternberg's (1981) and weighted averaging models such as Busemeyer's (1991), what makes it distinct from those models is the partition of contingency information into confirmatory and disconfirmatory instances. This leads to some predictions that are unique to the EE model. For example, the model predicts that when one kind of confirmatory information (e.g., cell $a$ ) is altogether absent from a set of instances, greater weight is given to the other kind (cell $d$ ). Similarly, if one kind of disconfirmatory information (e.g., cell $b$ ) is absent, then greater weight is given to the other kind (cell $c$ ). These predictions were supported by the results of two experiments in White (2003a).

The key question about the EE model concerns its capacity to predict results that are not predicted by the other leading models. White (2003a) showed that other models, including the $\Delta P$ rule and the power PC theory, do not predict the results just described. Tests of the unweighted version of Equation 2 have also provided discriminatory evidence (White, 2000b, 2002a, 2002b, 2002c, 2003a, 2003c).

These experiments shared a common strategy that made use of a pair of experimental designs. In one pair, $p C I$ values were varied and values of other variables were held constant. These variables include $\Delta P$ and its constituents, $a /(a+b)$ and $c /(c+d)$. They also include the base rate of occurrence of the effect, $p(\mathrm{E})=(a+c) /$ $(a+b+c+d)$, and the base rate of presence of the cause, $p(\mathrm{C})=(a+b) /(a+b+c+d)$. In the other pair, $\Delta P$ values were varied and values of $p \mathrm{CI}$ and other variables were held constant. It was consistently found that causal judgments tended to vary with $p \mathrm{CI}$ when $\Delta P$ and the other variables were held constant, and that they did not vary significantly with $\Delta P$ when $p C I$ and other variables were held constant. It was also shown that other models, including the power PC theory (Cheng, 1997), a weighted version of the $\Delta P$ rule (Lober $\&$ Shanks, 2000), and the Rescorla-Wagner model (Rescorla \& Wagner, 1972), failed to predict the observed tendencies for causal judgments to vary with $p$ CI values (White, 2000b, 2002b, 2002c, 2003a, 2003c).

These experiments therefore make a strong case for the unweighted $p$ CI rule as an account of asymptotic causal judgment. However, the design strategy of controlling other variables has some weaknesses. In particular, the requisite control cannot be achieved by comparing individual causal judgment problems. White (2000b, 2002a, 2002b, 2002c, 2003c) dealt with the need to hold other variables constant by comparing pairs of judgment problems. Individual members of each pair had different values of the other variables, but the sum of these values for each pair was constant.

This will be illustrated with one variable, $p(\mathrm{E})$. In White (2003c, Experiment 1), there were two pairs of judgment problems with different values of $p$ CI. In one pair, $p \mathrm{CI}=+.17$ in both problems, and in the other pair $p \mathrm{CI}=+.33$ in both problems. In the former pair, $p(\mathrm{E})=$ .58 in one member of the pair and .42 in the other. In the latter pair, $p(\mathrm{E})=.67$ in one member and .33 in the other. It can be seen that the sum of values of $p(\mathrm{E})=1$ for each pair, so $p(\mathrm{E})$ is held constant across the comparison between the pairs. However, the actual values of $p(\mathrm{E})$ differ between the pairs. Therefore, to be a valid test of the $p C I$ rule, the comparison requires the assumption that the relation between $p(\mathrm{E})$ and causal judgment is linear. In other words, changing $p(\mathrm{E})$ from .58 to .67 must have the same effect as changing $p(\mathrm{E})$ from .33 to .42 , because these are quantitatively equal changes. If this assumption does not hold, then the test of the $p$ CI rule is compromised.

A second weakness with this design is that it does not allow a systematic treatment of the relation between $p C I$ and other variables: The constraints imposed by the need to hold so many variables constant limit the range of comparisons between different values of $p \mathrm{CI}$ that can be conducted. This precludes the possibility of ascertaining how causal judgment varies over a wide and representative range of values of $p \mathrm{CI}$.

In addition, the unweighted version of the $p C I$ rule is not a completely accurate predictor of causal judgments. Significant effects occurred in the designs used by White (2000b, 2002a, 2002c, 2003c) in addition to those predicted by the unweighted version of the $p C I$ rule. These additional effects reflect unequal weightings of the four cells in causal judgment. In several studies, it has been found that, in terms of weights, $a>b>c>d$ (J. R. Anderson \& Sheu, 1995; Kao \& Wasserman, 1993; Levin, Wasserman, \& Kao, 1993; Mandel \& Lehman, 1998; Wasserman, Dorner, \& Kao, 1990). For the rule to make accurate predictions, these weights should be taken into account.

The present study was designed to address these issues and, thereby, to investigate a more systematic range of relations between $p \mathrm{CI}$ and other variables. The key variable for manipulations of $p \mathrm{CI}$ is $p(\mathrm{C})$, the base rate probability of the cause being present. It has been shown that when $p(\mathrm{C})=.5, p \mathrm{CI}$ values and $\Delta P$ values are identical (White, 2003c). This feature of the $p$ CI rule enables it to account for the many findings of close correspon- 
dence between causal judgments and $\Delta P$ values (see, e.g., J. R. Anderson \& Sheu, 1995; Wasserman, Chatlosh, \& Neunaber, 1983; Wasserman, Elek, Chatlosh, \& Baker, 1993; White, 2000a), because in those studies $p(\mathrm{C})=.5$ (White, 2003c). When $p(\mathrm{C}) \neq .5$, then $p \mathrm{CI}$ tends to diverge from $\Delta P$, but the manner in which it does so depends on the value of $a /(a+b)$ and, by implication, $c /(c+d)$ for a given value of $\Delta P$.

Figure 1 illustrates this. Panels A, C, and E show unweighted $p$ CI values plotted against values of $p(C)$ for dif- ferent values of $a /(a+b)$. Panel A shows this relation for $\Delta P=+.75$, panel $\mathrm{C}$ shows the relation for $\Delta P=+.5$, and panel $E$ shows the relation for $\Delta P=0$. The three panels show a similar pattern: As $a /(a+b)$ decreases, so the line relating $p \mathrm{CI}$ to $p(\mathrm{C})$ rotates clockwise around the point where $p(\mathrm{C})=.5$, at which value $p \mathrm{CI}=\Delta P$.

The counterparts to these three graphs - namely, panels B, D, and F of Figure 1 - show how this relation changes when a weighted version of $p C I$ is computed. For the purpose of constructing these panels of Figure 1,
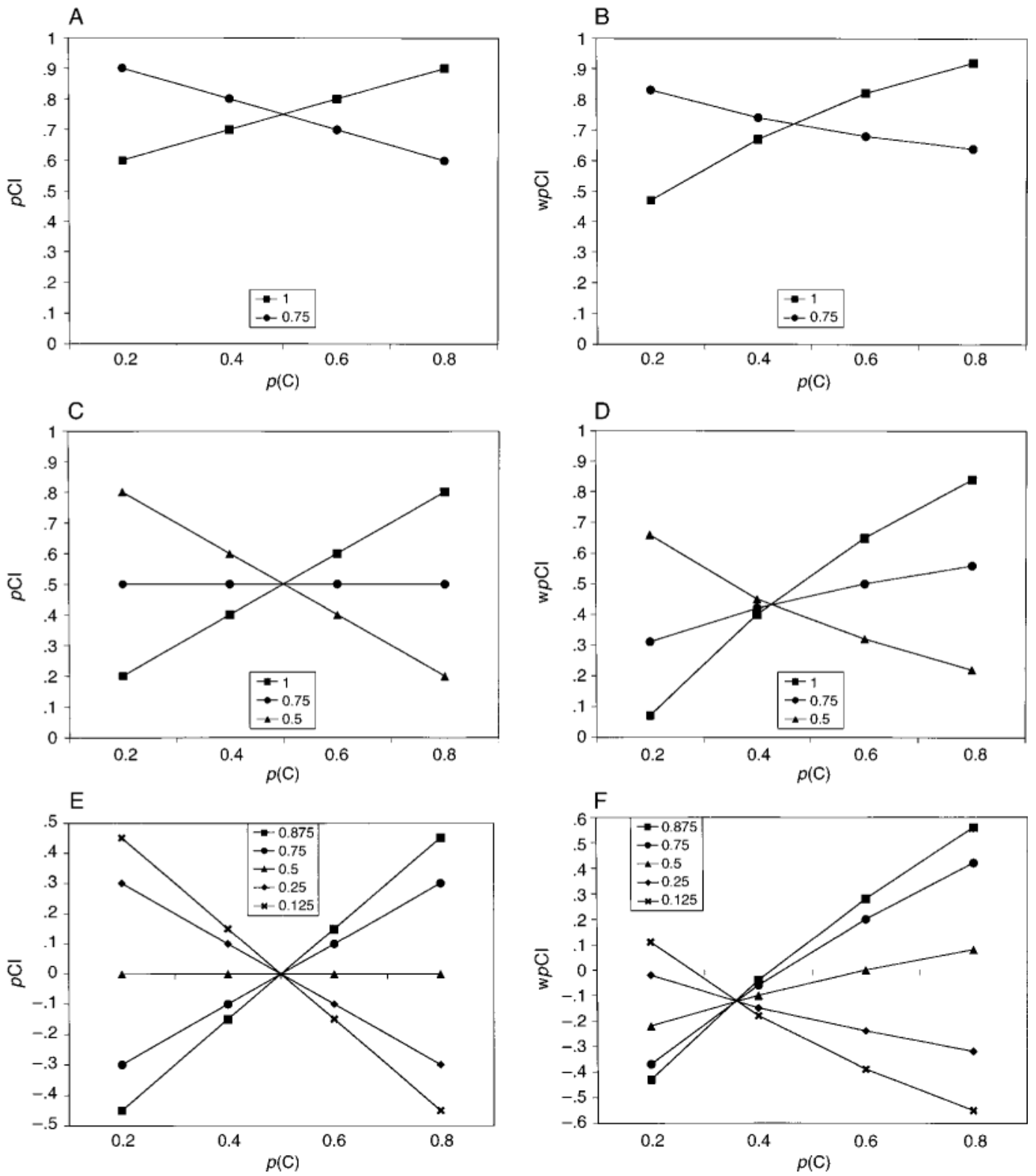

Figure 1. Values of unweighted $p C I$ and weighted $p C I$ at given values of $\Delta P$ for different combinations of $p(C)$ and $a /(a+b)$. Panels $A, C$, and $E$ show values of unweighted $p C I$ for $\Delta P=+.75,+.5$, and 0 , respectively. Panels $B, D$, and $F$ show values of weighted $p C I$ for $\Delta P=+.75,+.5$, and 0 , respectively. The key to each panel identifies values of $a /(a+b)$. 
the findings of White (2003b, Experiment 1, positive wording condition) were used. This experiment was chosen for two reasons. One was that in that study a method similar to that of the present study was used and was deemed most suitable on those grounds. The other reason was that in that study an orthogonal manipulation of frequencies in the four cells was employed, which enabled the weights of the four cells in causal judgment to be assessed by means of an effect size measure (Keppel, Saufley, \& Tokunaga, 1992). The weights obtained by White (2003b) were as follows: $\mathrm{w}_{a}=.72, \mathrm{w}_{b}=.53, \mathrm{w}_{c}=$ .51 , and $\mathrm{w}_{d}=.23$. These weights are similar to those obtained in other investigations of the cell weight inequality (J. R. Anderson \& Sheu, 1995; Kao \& Wasserman, 1993; Levin et al., 1993; Mandel \& Lehman, 1998; Wasserman et al., 1990).

Weighted $p C I$ values were computed using these weights, and the results are shown in Figure 1, panels B, $\mathrm{D}$, and F. Some differences between these and the counterpart panels A, C, and E are worthy of note. The general effect of the weighting is a counterclockwise rotation of the curves relating $p \mathrm{CI}$ to $p(\mathrm{C})$. For example, in panel B the curve for $a /(a+b)=1$ rises more steeply than the corresponding curve in panel $\mathrm{A}$, and the curve for $a /(a+b)=.75$ declines less steeply. The other noteworthy feature is that the junction between the curves tends to be shifted toward lower values of $p(\mathrm{C})$. This tendency is stronger for lower values of $\Delta P$ and is therefore particularly evident in panel $\mathrm{F}$, where the junction occurs at a point where $p(\mathrm{C})<.4$.

These panels constitute a set of predictions for causal judgment. Specifically, they predict how causal judgments for a given value of $\Delta P$ should differ as a function of changes in $p(C)$ and $a /(a+b)$. Experiment 1 was designed to test these predictions. The predictions of two other inductive rules for causal judgment will also be tested. One is the $\Delta P$ rule itself. As illustrated in Figure 1 , there are three separate designs in Experiment 1, and all judgment problems within each design have the same value of $\Delta P$. Clearly, if judgments are made in accordance with $\Delta P$ values, then there will be no significant differences between problems within designs, other than chance fluctuations.

The other inductive rule is the rule of causal judgment under the power PC theory. Cheng (1997) argued that people use contingency information to estimate the causal powers of things. Causal power is defined as the probability with which a cause produces its effect. To make this estimate, it is necessary to distinguish occurrences of the effect that are produced by the cause being judged from occurrences of the effect that are produced by other possible causes. If the distribution of other possible causes is unknown (but can be assumed to be independent of that of the cause being judged), their role can be estimated from the base rate of occurrence of the effect. In other words, the probability of occurrence of the effect when the cause being judged is absent constitutes an estimate of the causal power of other possible causes.
The causal power of the cause being judged can then be estimated by adjusting $\Delta P$ in light of the information about other possible causes. To be specific, causal power, $\mathrm{p}$, is estimated by Equation 3 when $\Delta P \geq 0$ and by Equation 4 when $\Delta P<0$.

$$
\begin{gathered}
\mathrm{p}=\frac{\Delta P}{1-[c /(c+d)]} \\
\mathrm{p}=\frac{\Delta P}{c /(c+d)}
\end{gathered}
$$

These equations hold when the candidate cause and the set of all other causes of the effect are not confounded: In this respect, they imply a natural sensitivity to basic principles of experimental design in causal reasoning. Predictions generated by these rules will be assessed in Experiment 1 .

\section{EXPERIMENT 1}

\section{Method}

Participants. The participants were 42 Cardiff University undergraduates of subjects other than psychology, who participated in return for cash payment. Forty-four students originally participated, but two were excluded prior to analysis because they had omitted one or more responses.

Stimulus materials. The initial written instructions to the participants read as follows:

\footnotetext{
Imagine that you are a botanist. You are interested in a plant called Brunnera macrophylla. Usually this plant has plain green leaves. But sometimes plants of this species have decorative red spots on their leaves. You have the idea that whether a plant has these red spots or not is determined by whether there is a certain chemical present in the soil or not. Some chemicals might cause the plant to have red spots; others might prevent it from having red spots. You are testing various possible chemicals to see whether they make a difference to the occurrence or nonoccurrence of red spots.

To do this you take a sample of plants and grow some of them in soil that has the chemical in it and some of them in soil that does not have the chemical in it. You then see whether each plant has red spots or not. So, for each chemical, you'll see information about several plants, showing whether the chemical was present in the soil or not and whether the plant had red spots or not.

You can have a look at the first test on the next page. As you will see, an alphabetic code for the name of the chemical is given at the top of the page. Under that there are three columns. The first one just gives a number to each plant in the test. The second one tells you whether the chemical was present or absent in the soil for that plant, and the third one tells you whether the plant had red spots or not.

Your task is to answer the following question which appears at the bottom of the page:

"To what extent did this chemical affect the occurrence of red spots in the brunnera plants?"

To answer this question write a number from -100 to +100 beside the statement. -100 means that the chemical very strongly prevented the plants from having red spots. +100 means that the chemical very strongly caused the plants to have red spots. 0 (zero) means that the chemical had no effect either way. The more strongly you think the chemical prevented the plants from having red spots the lower the number below zero you should put. The more strongly you think the chemical caused the plants to have red spots the higher the number above zero you should put.
}

There were 40 problems, 1 on each page. At the top of the page, a chemical was identified by two letters, which were different on each page. Beneath that were three columns in which information about 40 instances (plants) were reported. In the left column, the 
plant was identified with a number from 1 to 40 . The middle column was headed "chemical" and indicated, for each plant, whether the chemical was present or absent. The right column was headed "red spots" and indicated whether each plant had red spots ("yes") or not ("no"). Beneath the 40 instances was the question, "To what extent did this chemical affect the occurrence of red spots in the brunnera plants?"

Design. There were three separate experimental designs, each for one value of $\Delta P$. In each case, the independent variables were $p(\mathrm{C})$ and $a /(a+b)$. In each design, $p(\mathrm{C})$ was manipulated with four values: $.2, .4, .6$, and .8 . In the $\Delta P=+.75$ design, $a /(a+b)$ was manipulated with two values: 1.0 and .75 . In the $\Delta P=+.5$ design, $a /(a+b)$ was manipulated with three values: $1.0, .75$, and .5 . In the $\Delta P=0$ design, $a /(a+b)$ was manipulated with five values: .875 , $.75, .5, .25$, and .125 . These are the values that are plotted in relation to $p \mathrm{CI}$ in the respective panels of Figure 1. The numbers of judgmental problems in each design were therefore 8, 12, and 20, respectively, totaling 40 . In all cases, the manipulations were within subjects. Tables 2, 3, and 4 lay out cell frequencies for each combination of values of the independent variables in each design.

Procedure. The participants were tested singly or in groups of 2 or 3 in a large and comfortably furnished office. They were presented with a booklet containing the instructions for the task on the first page followed by the 40 judgmental problems in random order. A different random order was generated for each participant. Observations within each problem were also randomly ordered. The participants were invited to ask questions if anything in the in- structions was not clear. A few asked if they could write on the questionnaire and were told that they could. There were no other questions. When they had completed the questionnaire, they were thanked, paid, and debriefed as to the general purpose of the experiment.

\section{Results}

Results from three separate experimental designs are by nature a little complex, so I begin with a guide to the organization of this section. After formal description of the analyses, $F$ ratios and simple effects analyses of significant interactions in all three designs are presented in Tables 5 and 6, respectively. Means for individual problems within each design are presented in Tables 2, 3, and 4 and depicted in Figure 2. I assess the extent to which the trends in the figures match the predicted trends for the unweighted and weighted $p \mathrm{CI}$ rules. I then assess the predictions of the weighted $p$ CI rule for the main effects (see Tables 7 and 8). Finally, I report goodness of fit measures for the two versions of the $p \mathrm{CI}$ rule, the $\Delta P$ rule, and the power PC theory.

To summarize the main features of the results, the significant interactions conform to the patterns predicted by the weighted $p C I$ rule, and visual inspection of Figures 1

Table 2

Stimulus Materials, Predictions of Models, and Mean Causal Judgments, Experiment $1(\Delta P=.75$ Design)

\begin{tabular}{|c|c|c|c|c|c|c|c|c|c|}
\hline \multicolumn{4}{|c|}{ Cells } & \multicolumn{2}{|c|}{ Independent Variable } & \multicolumn{3}{|c|}{ Models } & \multirow[b]{2}{*}{ Mean Causal Judgment } \\
\hline$a$ & $b$ & $c$ & $d$ & $p(\mathrm{C})$ & $a /(a+b)$ & $p \mathrm{CI}$ & $\mathrm{w} p \mathrm{CI}$ & PPC & \\
\hline 8 & 0 & 8 & 24 & .2 & 1.0 & +.6 & +.47 & 1.0 & +48.26 \\
\hline 16 & 0 & 6 & 18 & .4 & 1.0 & +.7 & +.67 & 1.0 & +64.05 \\
\hline 24 & 0 & 4 & 12 & .6 & 1.0 & +.8 & +.82 & 1.0 & +74.81 \\
\hline 32 & 0 & 2 & 6 & .8 & 1.0 & +.9 & +.92 & 1.0 & +88.33 \\
\hline 6 & 2 & 0 & 32 & .2 & .75 & +.9 & +.83 & .75 & +79.79 \\
\hline 12 & 4 & 0 & 24 & .4 & .75 & +.8 & +.74 & .75 & +58.33 \\
\hline 18 & 6 & 0 & 16 & .6 & .75 & +.7 & +.68 & .75 & +58.38 \\
\hline 24 & 8 & 0 & 8 & .8 & .75 & +.6 & +.64 & .75 & +59.60 \\
\hline
\end{tabular}

Note $-p \mathrm{CI}$, unweighted $p \mathrm{CI}$; w $p \mathrm{CI}$, weighted $p \mathrm{CI}$; PPC, power PC theory. Each row describes a different judgment problem.

Table 3

Stimulus Materials, Predictions of Models, and Mean Causal Judgments, Experiment $1(\Delta P=.5$ Design $)$

\begin{tabular}{|c|c|c|c|c|c|c|c|c|c|}
\hline \multicolumn{4}{|c|}{ Cells } & \multicolumn{2}{|c|}{ Independent Variable } & \multicolumn{3}{|c|}{ Models } & \multirow[b]{2}{*}{ Mean Causal Judgment } \\
\hline$a$ & $b$ & $c$ & $d$ & $p(\mathrm{C})$ & $a /(a+b)$ & $p \mathrm{CI}$ & $\mathrm{w} p \mathrm{CI}$ & $\mathrm{PPC}$ & \\
\hline 8 & 0 & 16 & 16 & .2 & 1.0 & +.2 & +.07 & 1.0 & +30.29 \\
\hline 16 & 0 & 12 & 12 & .4 & 1.0 & +.4 & +.40 & 1.0 & +44.55 \\
\hline 24 & 0 & 8 & 8 & .6 & 1.0 & +.6 & +.65 & 1.0 & +60.48 \\
\hline 32 & 0 & 4 & 4 & .8 & 1.0 & +.8 & +.84 & 1.0 & +79.19 \\
\hline 6 & 2 & 8 & 24 & .2 & .75 & +.5 & +.31 & .67 & +19.81 \\
\hline 12 & 4 & 6 & 18 & .4 & .75 & +.5 & +.42 & .67 & +35.71 \\
\hline 18 & 6 & 4 & 12 & .6 & .75 & +.5 & +.50 & .67 & +43.24 \\
\hline 24 & 8 & 2 & 6 & .8 & .75 & +.5 & +.56 & .67 & +44.48 \\
\hline 4 & 4 & 0 & 32 & .2 & .5 & +.8 & +.66 & .5 & +37.45 \\
\hline 8 & 8 & 0 & 24 & .4 & .5 & +.6 & +.45 & .5 & +37.81 \\
\hline 12 & 12 & 0 & 16 & .6 & .5 & +.4 & +.32 & .5 & +19.76 \\
\hline 16 & 16 & 0 & 8 & .8 & .5 & +.2 & +.22 & .5 & +13.43 \\
\hline
\end{tabular}

Note $-p \mathrm{CI}$, unweighted $p \mathrm{CI}$; w $p \mathrm{CI}$, weighted $p \mathrm{CI}$; PPC, power PC theory. Each row describes a different judgment problem. 
Table 4

Stimulus Materials, Predictions of Models, and Mean Causal Judgments, Experiment 1 ( $\Delta \mathbf{P}=\mathbf{0}$ Design)

\begin{tabular}{|c|c|c|c|c|c|c|c|c|c|}
\hline \multicolumn{4}{|c|}{ Cells } & \multicolumn{2}{|c|}{ Independent Variable } & \multicolumn{3}{|c|}{ Models } & \multirow[b]{2}{*}{ Mean Causal Judgment } \\
\hline$a$ & $b$ & $c$ & $d$ & $p(\mathrm{C})$ & $a /(a+b)$ & $p \mathrm{CI}$ & $\mathrm{w} p \mathrm{CI}$ & PPC & \\
\hline 7 & 1 & 28 & 4 & .2 & .875 & -.45 & -.43 & 0 & -6.43 \\
\hline 14 & 2 & 21 & 3 & .4 & .875 & -.15 & -.04 & 0 & -3.14 \\
\hline 21 & 3 & 14 & 2 & .6 & .875 & +.15 & +.28 & 0 & +21.76 \\
\hline 28 & 4 & 7 & 1 & .8 & .875 & +.45 & +.56 & 0 & +40.69 \\
\hline 6 & 2 & 24 & 8 & .2 & .75 & -.30 & -.37 & 0 & -21.05 \\
\hline 12 & 4 & 18 & 6 & .4 & .75 & -.10 & -.06 & 0 & -1.21 \\
\hline 18 & 6 & 12 & 4 & 6 & .75 & +.10 & +.20 & 0 & +13.48 \\
\hline 24 & 8 & 6 & 2 & .8 & .75 & +.30 & +.42 & 0 & +35.02 \\
\hline 4 & 4 & 16 & 16 & .2 & .5 & .0 & -.22 & 0 & -2.05 \\
\hline 8 & 8 & 12 & 12 & .4 & .5 & .0 & -.10 & 0 & -2.17 \\
\hline 12 & 12 & 8 & 8 & 6 & .5 & .0 & .00 & 0 & +14.50 \\
\hline 16 & 16 & 4 & 4 & .8 & .5 & .0 & +.08 & 0 & +10.14 \\
\hline 2 & 6 & 8 & 24 & .2 & .25 & +.30 & -.02 & 0 & -19.43 \\
\hline 4 & 12 & 6 & 18 & .4 & .25 & +.10 & -.15 & 0 & -11.64 \\
\hline 6 & 18 & 4 & 12 & .6 & .25 & -.10 & -.24 & 0 & -23.19 \\
\hline 8 & 24 & 2 & 6 & .8 & .25 & -.30 & -.32 & 0 & -34.57 \\
\hline 1 & 7 & 4 & 28 & .2 & .125 & +.45 & +.11 & 0 & -16.79 \\
\hline 2 & 14 & 3 & 21 & .4 & .125 & +.15 & -.18 & 0 & -22.64 \\
\hline 3 & 21 & 2 & 14 & .6 & .125 & -.15 & -.39 & 0 & -34.95 \\
\hline 4 & 28 & 1 & 7 & .8 & .125 & -.45 & -.55 & 0 & -43.48 \\
\hline
\end{tabular}

Note $-p C I$, unweighted $p \mathrm{CI}$; w $p \mathrm{CI}$, weighted $p \mathrm{CI}$; PPC, power PC theory. Each row describes a different judgment problem.

and 2 also supports the contention that the weighted $p \mathrm{CI}$ rule provides a closer fit to observed means than the unweighted $p \mathrm{CI}$ rule does. The significant and nonsignificant main effects are also predicted by the weighted $p C I$ rule. The goodness-of-fit measures show that the weighted $p \mathrm{CI}$ rule is the best predictor of the observed tendencies. These results are now presented in more detail.

Data from the $\Delta P=+.75$ design were analyzed with a $4[p(\mathrm{C}): .2$ vs. .4 vs. .6 vs. .8$] \times 2[a /(a+b): 1.0$ vs. .75] within-subjects analysis of variance (ANOVA). Data from the $\Delta P=+.5$ design were analyzed with a 4 $[p(C): .2$ vs. .4 vs. .6 vs. .8$] \times 3[a /(a+b): 1.0$ vs. .75 vs. .5] within-subjects ANOVA. Data from the $\Delta P=0$ design were analyzed with a $4[p(\mathrm{C}): .2$ vs. .4 vs. .6 vs. $.8] \times 5[a /(a+b): .875$ vs. .75 vs. .5 vs. .25 vs. .125$]$ within-subjects ANOVA.

Table 6 shows results of post hoc paired comparisons carried out with the Newman-Keuls test. Mean causal judgments for individual judgmental problems are also shown in Table 6 , where they can be compared with the predictions of the unweighted and weighted $p C I$ rules, the $\Delta P$ rule, and the power $P C$ theory. Note that these predictions are ordinal in nature: They do not specify absolute values on the rating scale, but they do specify expected differences between different judgmental problems. For example, the $\Delta P$ rule predicts no differences between problems within the $\Delta P=.75$ design, but it does not predict any particular mean judgment for these problems.

The results are presented graphically in Figure 2. Panel A depicts the results for the $\Delta P=+.75$ design, panel $\mathrm{B}$, those for the $\Delta P=+.5$ design, and panel $\mathrm{C}$, those for the $\Delta P=0$ design. These will be compared with the corresponding panels in Figure 1.
In general, Figure 2 shows that observed means closely resemble the patterns predicted by the weighted $p C I$ rule. In each case, the curves for the higher values of $a /(a+b)$ rise more steeply than the curves for the lower values of $a /(a+b)$. In panels $\mathrm{A}$ and $\mathrm{B}$, the crossover point is displaced toward lower values of $p(C)$. These features are predicted by the weighted $p$ CI rule. In addition, in panel $\mathrm{C}$ of Figure 2 the curve for $a /(a+b)=.5$ shows higher means for the two higher values of $p(\mathrm{C})$ than for the two lower values, confirmed by simple effects analysis, and therefore approximately resembles the curve in panel $\mathrm{F}$ of Figure 1 and not the flat line in panel E of Figure 1. In this panel, it is hard to say whether there is a true crossover point, because the order of means for $p(\mathrm{C})=.2$ does not match the order shown in panel $\mathrm{F}$ of Figure 1. This is the main point of discrepancy between the predicted and observed tendencies, although it should be noted that there was no significant effect at this value of $p(\mathrm{C})$. In any case, if there is a crossover point it is certainly displaced toward lower values of $p(\mathrm{C})$, as in panel $\mathrm{F}$ of Figure 1.

Table 5

$F$ Ratios, Experiment 1

\begin{tabular}{llrrrc}
\hline Design & Effect & \multicolumn{1}{c}{$F$} & \multicolumn{1}{c}{$d f$} & \multicolumn{1}{c}{$M S_{\mathrm{e}}$} & \multicolumn{1}{c}{$p$} \\
\hline$\Delta P=+.75$ & $p(\mathrm{C})$ & 4.13 & 3,123 & 610.86 & $<.01$ \\
& $a /(a+b)$ & 3.85 & 1,41 & 510.55 & n.s. \\
& Interaction & 26.15 & 3,123 & 543.02 & $<.001$ \\
$\Delta P=+.50$ & $p(\mathrm{C})$ & 5.30 & 3,123 & $1,157.25$ & $<.01$ \\
& $a /(a+b)$ & 20.28 & 2,82 & $1,512.82$ & $<.001$ \\
& Interaction & 13.50 & 6,246 & 901.78 & $<.001$ \\
$\Delta P=0$ & $p(\mathrm{C})$ & 6.41 & 3,123 & $1,419.29$ & $<.001$ \\
& $a /(a+b)$ & 36.12 & 4,164 & $1,699.67$ & $<.001$ \\
& Interaction & 11.40 & 12,492 & $1,055.34$ & $<.001$ \\
\hline
\end{tabular}


Table 6

Simple Effects Analyses of Interactions, Experiment 1

\begin{tabular}{clrll}
\hline Design & \multicolumn{1}{c}{ Effect } & \multicolumn{1}{c}{$F$} & \multicolumn{1}{c}{$p$} & \multicolumn{1}{c}{ Differences } \\
\hline$\Delta P=+.75$ & $p(\mathrm{C})=.2$ & 25.24 & $<.001$ & $a /(a+b) .75>1.0$ \\
& $p(\mathrm{C})=.4$ & 1.08 & n.s. & \\
& $p(\mathrm{C})=.6$ & 14.89 & $<.001$ & $a /(a+b) .75<1.0$ \\
& $p(\mathrm{C})=.8$ & 58.41 & $<.001$ & $a /(a+b) .75<1.0$ \\
& $a /(a+b)=1.0$ & 20.26 & $<.001$ & $p(\mathrm{C}) .2<.4<.6<.8$ \\
& $a /(a+b)=.75$ & 8.33 & $<.001$ & $p(\mathrm{C}) .2>.4, .6$, and .8 \\
$\Delta P=+.50$ & $p(\mathrm{C})=.2$ & 2.65 & n.s. & \\
& $p(\mathrm{C})=.4$ & 0.90 & n.s. & \\
& $p(\mathrm{C})=.6$ & 14.04 & $<.001$ & $a /(a+b) .5<.75<1.0$ \\
& $p(\mathrm{C})=.8$ & 62.34 & $<.001$ & $a /(a+b) .5<.75<1.0$ \\
& $a /(a+b)=1.0$ & 23.23 & $<.001$ & $p(\mathrm{C}) .2$ and $.4<.6<.8$ \\
& $a /(a+b)=.75$ & 6.58 & $<.001$ & $p(\mathrm{C}) .2<.6$ and .8 \\
& $a /(a+b)=.5$ & 4.84 & $<.001$ & $p(\mathrm{C}) .2$ and $.4>.8$ \\
& $p(\mathrm{C})=.2$ & 1.90 & $\mathrm{n} . \mathrm{s}$. & \\
$p(\mathrm{C})=.4$ & 3.80 & $<.01$ & No differences \\
& $p(\mathrm{C})=.6$ & 22.04 & $<.001$ & $a /(a+b) .125$ and $.25<.5, .75$, and .875 \\
& $p(\mathrm{C})=.8$ & 55.85 & $<.001$ & $a /(a+b) .125$ and $.25<.5<.75<.875$ \\
& $a /(a+b)=.875$ & 12.70 & $<.001$ & $p(\mathrm{C}) .2$ and $.4<.6<.8$ \\
& $a /(a+b)=.75$ & 15.92 & $<.001$ & $p(\mathrm{C}) .2<.6$ and $.8 ; .4<.8$ \\
& $a /(a+b)=.5$ & 5.39 & $<.01$ & .2, and $.4<.6$ and .8 \\
& $a /(a+b)=.25$ & 4.31 & $<.01$ & $.2, .4$, and $.6>.8$ \\
& $a /(a+b)=.125$ & 5.63 & $<.01$ & $.2, .4$ and $.6>.8$ \\
& & & &
\end{tabular}

Overall, the pattern of interactions and simple effects analyses reported in Table 6 confirms the visual impression that the results presented in Figure 2 resemble the predictions of the weighted $p \mathrm{CI}$ rule better than those of the unweighted rule, and in most respects the match between predicted and observed tendencies for the weighted $p C$ I rule was indeed close.

The unweighted $p$ CI rule predicts significant interactions between $p(\mathrm{C})$ and $a /(a+b)$, as is shown in panels $\mathrm{A}, \mathrm{C}$, and $\mathrm{E}$ of Figure 1. However, it does not predict main effects of either variable: This is shown by the symmetrical patterns in each of those three panels. The weighted $p C I$ rule, however, does predict the significant main effects of $p(\mathrm{C})$ and $a /(a+b)$. This can be shown by computing mean weighted $p \mathrm{CI}$ values for the problems that comprise each value of each independent variable and comparing them with the observed main effects. This is done in Table 7 for $a /(a+b)$ and in Table 8 for $p(\mathrm{C})$.

Consider Table 7 first. When $\Delta P=+.75$, both values of $a /(a+b)$ have the same mean weighted $p \mathrm{CI}$, which amounts to a prediction of no difference between the means, and this was indeed the only main effect that failed to be statistically significant. When $\Delta P=+.5$, mean weighted $p C I$ values tend to decrease as $a /(a+b)$ decreases, and this is matched by a statistically significant decreasing trend in the observed means. The same is the case for $\Delta P=0$. Turning to Table 8 , at all three values of $\Delta P$ mean weighted $p C I$ values tend to increase as $p(\mathrm{C})$ increases, and in all three cases the observed means also show statistically significant tendencies to increase as $p(\mathrm{C})$ increases. The mean for $p(\mathrm{C})=.2$ when $\Delta P=+.75$ is anomalous in this respect, but was not significantly different from the mean for $p(\mathrm{C})=.4$. The weighted $p$ CI rule can therefore account for all the observed tendencies in the results.

Overall goodness of fit between predictions and results was assessed by correlating predicted values for unweighted and weighted versions of $p \mathrm{CI}$, the $\Delta P$ rule, and $p$ with mean causal judgments using the Pearson correlation coefficient. Results are shown in Table 9. The table shows that the weighted version of $p$ CI was the best predictor in all four comparisons, but the unweighted version of $p C I$ also fitted the observed means better than $\Delta P$ and $p$ in all four comparisons.

\section{Discussion}

First, the main features of the results will be summarized. Overall, the weighted version of the $p$ CI rule was a good predictor of the observed judgment tendencies, as is shown primarily by the similarity between the three panels of Figure 2 and panels B, D, and F of Figure 1. The weighted $p \mathrm{CI}$ rule, unlike the unweighted $p \mathrm{CI}$ rule, predicted the main effects obtained in each analysis. Goodness-of-fit measures show that the weighted $p \mathrm{CI}$ rule consistently outperformed the unweighted $p \mathrm{CI}$ rule, which in turn was a consistently better predictor than $\Delta P$ and the power PC theory.

One possible defense of the poor performance of the power PC theory is that the dependent measure is inappropriate as a test of it. The participants were asked to judge to what extent the cause produced the effect. This measure was chosen as an alternative to measures of judged likelihood (e.g., "How likely do you think it is that the cause produces the effect?") on the grounds that such measures may conflate causal judgment with a rating of subjective confidence. Clearly, if judgments reflected a feeling of confidence, then the results would 

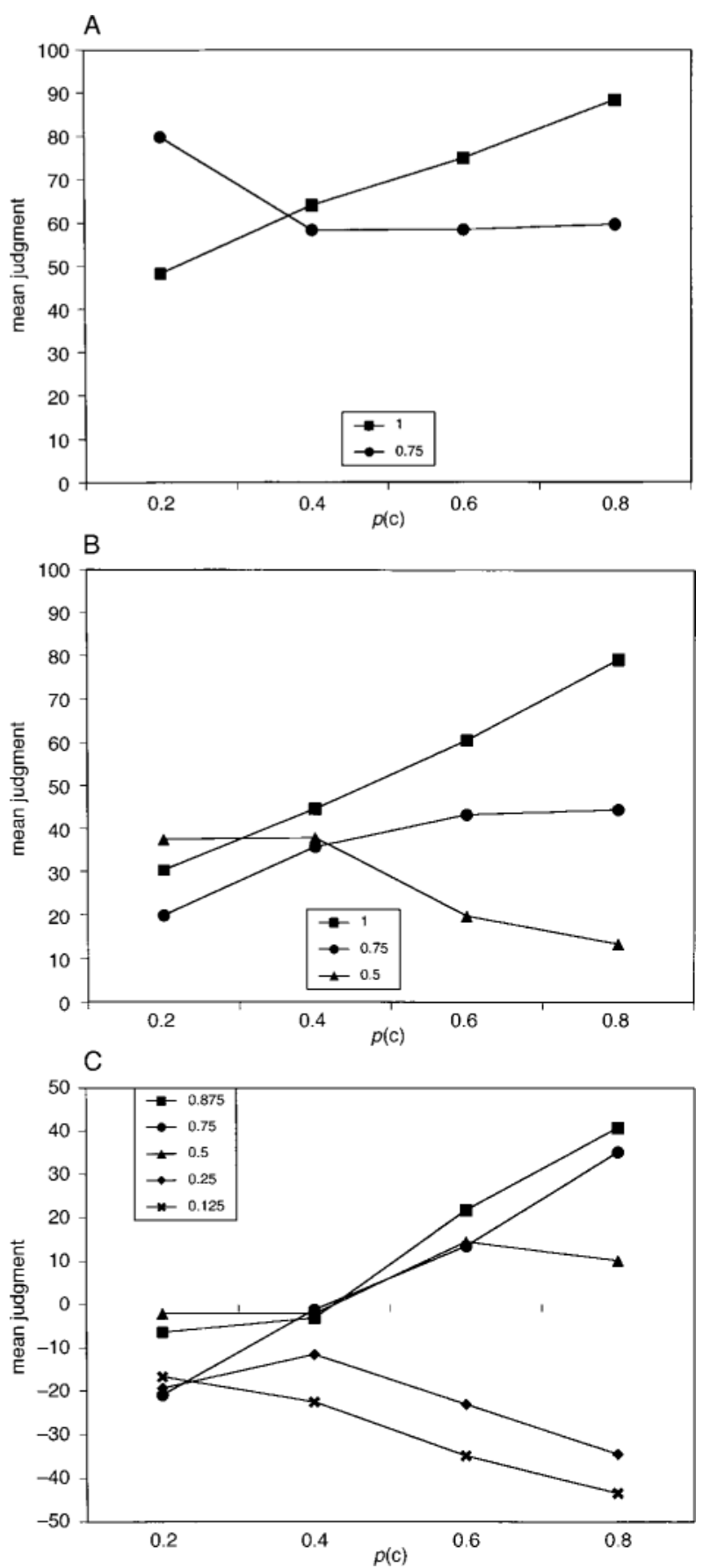

Figure 2. Mean causal judgments, Experiment 1. Panels A, B, and $C$ show means for $\Delta P=+.75,+.5$, and 0 , respectively. The key to each panel identifies values of $a /(a+b)$.

not be valid tests of an assessment of power. The present measure was selected precisely to be a measure of power.

It could be argued that the dependent measure is inappropriately worded, because the power PC theory measures the likelihood that an effect would occur given that a causal candidate is present and no other alternative causes are present. In fact, as was explained in the introduction, the power PC theory measures the probability with which a cause produces an effect. It does so not by requiring that other causes be absent, but by stipulating how their contribution can be distinguished from that of the cause being judged. This does require some assumptions, principally the assumption that the distribution of other causes is independent of the distribution of the cause being judged. But these assumptions are not compromised by the manipulations used in Experiment 1: Other causes are as likely to be independent of the cause being judged when $p(\mathrm{C})$ is high as when it is low. Cheng (1997) derived predictions from the power PC theory for numerous studies of causal judgment in which use was made of measures with similar wording to that used here, and she did not argue that the measures were inappropriately worded.

Some examples of studies cited in Cheng's (1997) review can be given. J. R. Anderson and Sheu (1995, Experiment 1) asked participants to judge "how likely the drug used in that hospital is the cause of side effects in patients" (p. 514). In Baker, Berbrier, and ValléeTourangeau (1989), participants were asked to estimate the effectiveness of shells in causing tanks to explode. Kao and Wasserman (1993) asked participants to rate "the value of the fertilizer in promoting the Lanyu to bloom" (p. 1369). Shanks (1987) had participants "judge the extent to which pressing the SPACE BAR causes the triangle to light up" (p. 153). In Wasserman et al. (1993), participants were asked to judge "the degree to which your tapping of the telegraph key affected the occurrence of the white light" (p. 176). More recently, in Lober and Shanks (2000), a study designed to test the predictions of the power PC theory, participants were asked to "estimate the degree to which the chemical causes mutations" (p. 202).

A likelihood judgment was used by J. R. Anderson and Sheu (1995), but in all the other studies a causal judgment question was posed similar in essential features to the question used in the present research. If measures worded in this way were not to be accepted as proper tests of the theory, then most findings in this literature would lie outside its scope and much of the evidence assessed by Cheng (1997) as supportive of the theory would be ruled out, which would be a most unsatisfactory state of affairs for the theory.

Developing the objection further, it could be argued that judgments on a measure of strength could be influenced by the prevalence of the cause: A more prevalent cause might be judged stronger than a less prevalent one, because it accounts for more of the occurrences of the effect. On these grounds, it could be argued that the measure does not provide a valid test of the power PC theory because prevalence effects are theoretically distinct from effects of causal power. Prevalence is captured by $p(\mathrm{C})$; therefore, a prevalence effect would show itself as a main effect of that factor. The results did show main effects of $p(\mathrm{C})$ and were predicted by the weighted $p$ CI rule. How- 
Table 7

Mean Weighted $p$ CI Values Compared With Observed Means for Main Effects of $a /(a+b)$, Experiment 1

\begin{tabular}{cccr}
\hline & & \multicolumn{2}{c}{ Means } \\
\cline { 3 - 4 }$\Delta P$ & $a /(a+b)$ & Observed & w $p$ CI \\
\hline+.75 & 1.0 & 68.86 & .72 \\
+.50 & .75 & 64.02 & .72 \\
& 1.0 & 53.62 & .49 \\
& .75 & 35.81 & .45 \\
0 & .5 & 27.11 & .41 \\
& .875 & 13.22 & .09 \\
& .75 & 6.56 & .05 \\
& .5 & 5.11 & -.06 \\
& .25 & -22.21 & -.18 \\
& .125 & -29.46 & -.25 \\
\hline
\end{tabular}

Table 8

Mean Weighted $p$ CI Values Compared With Observed Means for Main Effects of $p(c)$, Experiment 1

\begin{tabular}{cccc}
\hline & & \multicolumn{2}{c}{ Means } \\
\cline { 3 - 4 }$\Delta P$ & $p(\mathrm{C})$ & Observed & $\mathrm{w} p \mathrm{CI}$ \\
\hline+.75 & .2 & 64.02 & .65 \\
& .4 & 61.19 & .70 \\
& .6 & 66.60 & .75 \\
+.50 & .8 & 73.96 & .78 \\
& .2 & 29.18 & .35 \\
& .4 & 39.36 & .42 \\
0 & .6 & 41.16 & .49 \\
& .8 & 45.70 & .54 \\
& .2 & -13.15 & -.19 \\
& .4 & -8.16 & -.11 \\
& .6 & -1.68 & -.03 \\
& .8 & 1.56 & .04 \\
\hline
\end{tabular}

ever, the main findings claimed as support for the $p \mathrm{CI}$ rule were not those main effects but interactions between $p(\mathrm{C})$ and $a /(a+b)$. These interactions show that, at some values of $a /(a+b)$, mean judgments decline significantly as $p(\mathrm{C})$ increases. Two examples are the trends for $a /(a+b)=.25$ and .125 when $\Delta P=0$ (panel $\mathrm{C}$ of Figure 2). This is the opposite of a prevalence effect. Therefore, it can be concluded that the measure of strength is not confounded by prevalence.

The weights used to compute the predictions for the weighted $p$ CI rule were derived by a priori decision from a different previous experiment. It is of course possible that a different set of weights could be found that would generate an even more precise fit to the observed tendencies. However, the match between the mean tendencies of the participants in this experiment and predictions derived from weights found in a different experiment with different participants is sufficiently close to indicate that those weights have some general value as predictors.

\section{EXPERIMENT 2}

The three variables $p(\mathrm{C}), a /(a+b)$, and $\Delta P$ can all be varied independently. In Experiment 1, the predictions of the $p$ CI rule were tested for the case in which $p(C)$ and $a /(a+b)$ vary and $\Delta P$ is held constant. It is also important to show that the $p$ CI rule can predict what happens when $p(C)$ and $\Delta P$ vary and $a /(a+b)$ is held constant. This is the aim of Experiment 2. In this case, because $\Delta P$ is varied, one would expect both the $\Delta P$ rule and the power $\mathrm{PC}$ theory to predict judgments to some degree. The main means of differentiating between these rules and the $p \mathrm{CI}$ rule is the $p(\mathrm{C})$ manipulation. The $p \mathrm{CI}$ rule predicts effects of this manipulation, but the $\Delta P$ rule does not, so the results section will focus mainly on this comparison.

Figure 3 lays out the predictions of the $p$ CI rule in graphical form. Values of $p \mathrm{CI}$ are plotted against values of $\Delta P$ for different values of $p(C)$ while $a /(a+b)$ is held constant. Panels A, C, and E of this figure show unweighted $p$ CI values for $a /(a+b)=.75, .5$, and .25 , respectively. Panels $\mathrm{B}, \mathrm{D}$, and $\mathrm{F}$ show weighted $p \mathrm{CI}$ values computed with the same cell weights as in Experiment 1 for $a /(a+b)=.75, .5$, and .25 , respectively.

These graphs show that, when $p(\mathrm{C})=.5$, unweighted $p C I$ values match $\Delta P$ values exactly. As $p(C)$ increases from .2 to .8 , there is a clockwise rotation of the function around a pivotal point, the value of which changes with changes in $a /(a+b)$. The graphs showing weighted $p \mathrm{CI}$ values are broadly similar, although in each case the pivotal point of the rotation is displaced toward a lower value of $\Delta P$. Experiment 2 was designed to test these predictions.

\section{Method}

The details of the method were as in Experiment 1, with the following exceptions. The participants were 42 undergraduate students of subjects other than psychology, who participated in return for cash payment. None had participated in Experiment 1.

There were three separate experimental designs, each for one value of $a /(a+b)$. In each case, the independent variables were $p(\mathrm{C})$ and $\Delta P$. In each design, $p(\mathrm{C})$ was manipulated with 3 values: $.2, .5$, and .8 . In the $a /(a+b)=.75$ design, $\Delta P$ was manipulated with 5 values: $+.75,+.5,+.25,0$, and -.25 . In the $a /(a+b)=.5$ design, $\Delta P$ was manipulated with 5 values: $+.5,+.25,0,-.25$, and -.5 . In the $a /(a+b)=.25$ design, $\Delta P$ was manipulated with 5 values: $+.25,0,-.25,-.5$, and -.75 . These are the values that are plotted in relation to $p \mathrm{CI}$ in the respective panels of Figure 2 . The number of judgmental problems was therefore 15 in each design, totaling 45. Tables 10, 11, and 12 lay out cell frequencies for each combination of values of the independent variables in each design.

\section{Results}

This Results section is organized in a similar way to that of Experiment 1. To summarize the main results, comparison between Figures 3 and 4 shows that the sig-

Table 9

Correlations Between Predicted and Observed Mean Causal Judgments, Experiment 1

\begin{tabular}{ccccr}
\hline & \multicolumn{4}{c}{ Model } \\
\cline { 2 - 5 }$\Delta P$ & $p \mathrm{CI}$ & $\mathrm{w} p \mathrm{CI}$ & $\Delta P$ & $\mathrm{PPC}$ \\
\hline+.75 & +.86 & +.93 & .00 & +.19 \\
+.50 & +.66 & +.82 & .00 & +.63 \\
0 & +.55 & +.87 & .00 & .00 \\
$\mathrm{All}$ & +.87 & +.95 & +.83 & +.83 \\
\hline
\end{tabular}


nificant interactions were generally better predicted by the weighted $p \mathrm{CI}$ rule than by the unweighted $p \mathrm{CI}$ rule. Main effects of the $p(\mathrm{C})$ manipulation were predicted by the weighted $p C I$ rule but not by the $\Delta P$ rule or by the power PC theory. The same was the case for three significant interactions between $p(\mathrm{C})$ and $\Delta P$.

In all three designs, data were analyzed with a $3[p(\mathrm{C})$ : .2 vs. .5 vs. . 8$] \times 5(\Delta P)$ within-subjects ANOVA. $F$ ratios are reported in Table 13. Results of simple effects analyses on the significant interactions are reported in Table 14. This table shows results of post hoc paired comparisons carried out with the Newman-Keuls test. Mean causal judgments for individual problems are shown in Tables 10, 11 , and 12 , where they can be compared with the predictions of the unweighted and weighted $p C I$ rules, the $\Delta P$ rule, and the power PC theory. The results are presented graphically in Figure 4. Panel A depicts the results for the $a /(a+b)=.75$ design, panel B for the $a /(a+b)=.5$ design, and panel $\mathrm{C}$ for the $a /(a+b)=.25$ design. The results will be compared with the corresponding panels in Figure 3.

In general, a comparison between Figures 3 and 4 shows that the observed means resembled the means predicted by the $p C I$ rule. In particular, at high values of $\Delta P$
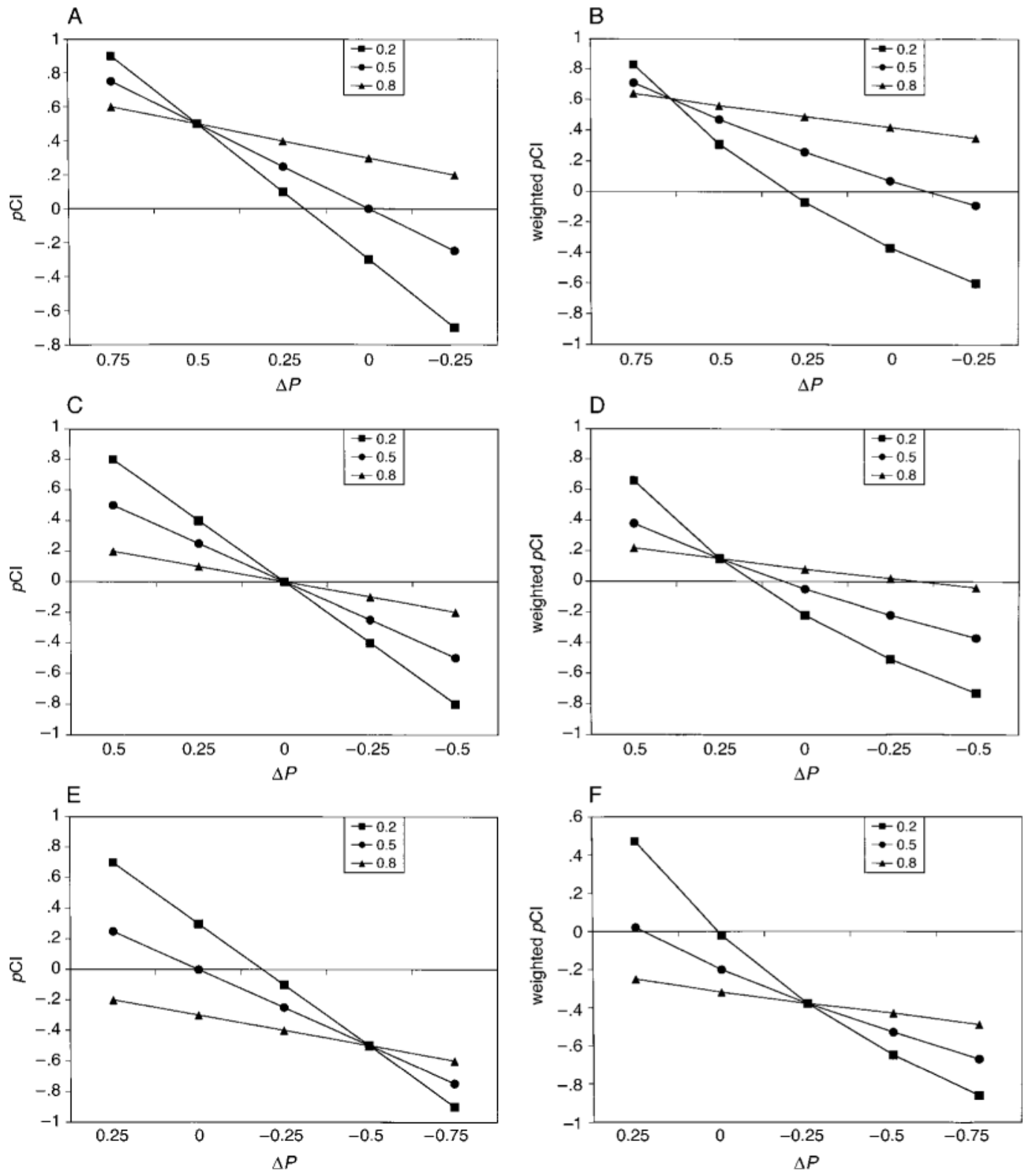

Figure 3. Values of unweighted $p C I$ and weighted $p C I$ at given values of $a /(a+b)$ for different combinations of $p(C)$ and $\Delta P$. Panels $A, C$, and $E$ show values of unweighted $p C I$ for $a /(a+b)=.75, .5$, and .25 , respectively. Panels B, D, and F show values of weighted $p C I$ for $a /(a+b)=.75, .5$, and .25 , respectively. The key to each panel identifies values of $p(C)$. 
Table 10

Stimulus Materials, Predictions of Models, and Mean Causal Judgments, Experiment 2 $[a /(a+b)=.50$ Design $]$

\begin{tabular}{|c|c|c|c|c|c|c|c|c|c|}
\hline \multicolumn{4}{|c|}{ Cells } & \multicolumn{2}{|c|}{ Independent Variable } & \multicolumn{3}{|c|}{ Models } & \multirow[b]{2}{*}{ Mean Causal Judgment } \\
\hline$a$ & $b$ & $c$ & $\bar{d}$ & $\overline{p(C)}$ & $\Delta P$ & $p \mathrm{CI}$ & $\mathrm{wpCI}$ & PPC & \\
\hline 6 & 2 & 0 & 32 & .2 & +.75 & +.90 & +.83 & +.75 & +68.36 \\
\hline 6 & 2 & 8 & 24 & .2 & +.50 & +.50 & +.31 & +.67 & +22.81 \\
\hline 6 & 2 & 16 & 16 & .2 & +.25 & +.10 & -.07 & +.50 & +13.14 \\
\hline 6 & 2 & 24 & 8 & .2 & .00 & -.30 & -.37 & .00 & -10.55 \\
\hline 6 & 2 & 32 & 0 & .2 & -.25 & -.70 & -.60 & -.25 & -34.07 \\
\hline 15 & 5 & 0 & 20 & .5 & +.75 & +.71 & +.75 & +.75 & +63.02 \\
\hline 15 & 5 & 5 & 15 & .5 & +.50 & +.47 & +.50 & +.67 & +52.14 \\
\hline 15 & 5 & 10 & 10 & .5 & +.25 & +.26 & +.25 & +.50 & +28.81 \\
\hline 15 & 5 & 15 & 5 & .5 & .00 & +.07 & .00 & .00 & +6.79 \\
\hline 15 & 5 & 20 & 0 & .5 & -.25 & -.09 & -.25 & -.25 & -1.74 \\
\hline 24 & 8 & 0 & 8 & .8 & +.75 & +.64 & +.60 & +.75 & +65.62 \\
\hline 24 & 8 & 2 & 6 & .8 & +.50 & +.56 & +.50 & +.67 & +54.36 \\
\hline 24 & 8 & 4 & 4 & .8 & +.25 & +.49 & +.40 & +.50 & +44.79 \\
\hline 24 & 8 & 6 & 2 & .8 & .00 & +.42 & +.30 & .00 & +39.29 \\
\hline 24 & 8 & 8 & 0 & .8 & -.25 & +.35 & +.20 & -.25 & +7.12 \\
\hline
\end{tabular}

Note $-p$ CI, unweighted $p C I$; $p$ CI, weighted $p C I$; PPC, power PC theory. Each row describes a different judgment problem.

Table 11

Stimulus Materials, Predictions of Models, and Mean Causal Judgments, Experiment 2 $[a /(a+b)=.75$ Design $]$

\begin{tabular}{|c|c|c|c|c|c|c|c|c|c|}
\hline \multicolumn{4}{|c|}{ Cells } & \multicolumn{2}{|c|}{ Independent Variable } & \multicolumn{3}{|c|}{ Models } & \multirow[b]{2}{*}{ Mean Causal Judgment } \\
\hline$a$ & $b$ & $c$ & $d$ & $p(\mathrm{C})$ & $\Delta P$ & $p \mathrm{CI}$ & $\mathrm{w} p \mathrm{CI}$ & $\mathrm{PPC}$ & \\
\hline 4 & 4 & 0 & 32 & .2 & +.50 & +.66 & +.80 & +.50 & +42.71 \\
\hline 4 & 4 & 8 & 24 & .2 & +.25 & +.15 & +.40 & +.33 & +13.64 \\
\hline 4 & 4 & 16 & 16 & .2 & .00 & -.22 & .00 & .00 & -2.90 \\
\hline 4 & 4 & 24 & 8 & .2 & -.25 & -.51 & -.40 & -.33 & -21.38 \\
\hline 4 & 4 & 24 & 8 & .2 & -.50 & -.73 & -.80 & -.50 & -51.00 \\
\hline 10 & 10 & 0 & 20 & .5 & +.50 & +.38 & +.50 & +.50 & +31.26 \\
\hline 10 & 10 & 5 & 15 & .5 & +.25 & +.15 & +.25 & +.33 & +16.29 \\
\hline 10 & 10 & 10 & 10 & .5 & .00 & -.05 & .00 & .00 & +0.50 \\
\hline 10 & 10 & 15 & 5 & .5 & -.25 & -.22 & -.25 & -.33 & -3.21 \\
\hline 10 & 10 & 20 & 0 & .5 & -.50 & -.37 & -.50 & -.50 & -35.12 \\
\hline 16 & 16 & 0 & 8 & .8 & +.50 & +.22 & +.20 & +.50 & +29.98 \\
\hline 16 & 16 & 2 & 6 & .8 & +.25 & +.15 & +.10 & +.33 & +18.87 \\
\hline 16 & 16 & 4 & 4 & .8 & .00 & +.08 & .00 & .00 & +11.19 \\
\hline 16 & 16 & 6 & 2 & .8 & -.25 & +.02 & -.10 & -.33 & -1.98 \\
\hline 16 & 16 & 8 & 0 & .8 & -.50 & -.04 & -.20 & -.50 & +4.10 \\
\hline
\end{tabular}

Note $-p C I$, unweighted $p C I$; w $p C I$, weighted $p C I$; PPC, power PC theory. Each row describes a different judgment problem.

means tended to decrease as $p(\mathrm{C})$ increased, and at lower values of $\Delta P$ there was an increasing tendency for means to increase as $p(\mathrm{C})$ increased. In this experiment, predictions for the unweighted and weighted versions of $p C I$ are more similar than was the case in Experiment 1, so it is not so easy to decide which version gives the better fit to the data. The clearest differentiating feature is the location of the crossover point, which was consistently further to the left in the graphs for weighted $p$ CI. In this regard, the results tend to favor weighted $p \mathrm{CI}$.

It is perhaps needless to say that both versions of $p \mathrm{CI}$ predict the main effect of the $\Delta P$ manipulation: It is evident in Figure 3 that mean values of both unweighted $p C I$ and weighted $p \mathrm{CI}$ tended to decrease as $\Delta P$ decreases. However, the main interest of this experiment, as far as models of causal judgment are concerned, lies with the $p(\mathrm{C})$ manipulations. The $\Delta P$ rule predicts no effects of $p(C)$. The same is true of the power PC theory. This can be seen in Tables 10, 11, and 12. In each table, the power PC theory always generates the same value for a given value of $\Delta P$, regardless of the value of $p(\mathrm{C})$. Mean values of unweighted and weighted $p$ CI for values of $p(\mathrm{C})$ are shown in Table 15. When $a /(a+b)=.75$, mean judgments tended to increase as $p(C)$ increased, and both the unweighted and weighted versions of $p C I$ predict this tendency. When $a /(a+b)=.5$, a similar tendency was evident in the observed means, but it is predicted only by the weighted version of $p$ CI: Mean values of the unweighted version are the same for each value of $p(C)$. When $a /(a+b)=.25$, there was no significant difference between the means. Both versions of $p \mathrm{CI}$ predict a tendency for means to decrease as $p(\mathrm{C})$ increases, but 
Table 12

Stimulus Materials, Predictions of Models, and Mean Causal Judgments, Experiment 2 $[a /(a+b)=.25$ Design $]$

\begin{tabular}{|c|c|c|c|c|c|c|c|c|c|}
\hline \multicolumn{4}{|c|}{ Cells } & \multicolumn{2}{|c|}{ Independent Variable } & \multicolumn{3}{|c|}{ Models } & \multirow[b]{2}{*}{ Mean Causal Judgment } \\
\hline$a$ & $b$ & $c$ & $d$ & $p(\mathrm{C})$ & $\Delta P$ & $p \mathrm{CI}$ & $\mathrm{w} p \mathrm{CI}$ & $\mathrm{PPC}$ & \\
\hline 2 & 6 & 0 & 32 & .2 & +.25 & +.47 & +.70 & +.25 & +12.79 \\
\hline 2 & 6 & 8 & 24 & .2 & .00 & -.02 & +.30 & .00 & -3.24 \\
\hline 2 & 6 & 16 & 16 & .2 & -.25 & -.38 & -.10 & -.50 & -27.55 \\
\hline 2 & 6 & 24 & 8 & .2 & -.50 & -.65 & -.50 & -.67 & -52.76 \\
\hline 2 & 6 & 32 & 0 & .2 & -.75 & -.86 & -.90 & -.75 & -78.83 \\
\hline 5 & 15 & 0 & 20 & .5 & +.25 & +.02 & +.25 & +.25 & +6.14 \\
\hline 5 & 15 & 5 & 15 & .5 & .00 & -.02 & .00 & .00 & -1.79 \\
\hline 5 & 15 & 10 & 10 & .5 & .25 & -.38 & -.25 & -.50 & -14.05 \\
\hline 5 & 15 & 15 & 5 & .5 & -.50 & -.53 & -.50 & -.67 & -46.98 \\
\hline 5 & 15 & 20 & 0 & .5 & -.75 & -.67 & -.75 & -.75 & -64.60 \\
\hline 8 & 24 & 0 & 8 & .8 & +.25 & -.25 & -.20 & +.25 & -1.48 \\
\hline 8 & 24 & 2 & 6 & .8 & .00 & -.32 & -.30 & .00 & -13.81 \\
\hline 8 & 24 & 4 & 4 & .8 & -.25 & -.38 & -.40 & -.50 & -21.26 \\
\hline 8 & 24 & 6 & 2 & .8 & -.50 & -.43 & -.50 & -.67 & -36.36 \\
\hline 8 & 24 & 8 & 0 & .8 & -.75 & -.49 & -.60 & -.75 & -56.67 \\
\hline
\end{tabular}

Note $-p \mathrm{CI}$, unweighted $p \mathrm{CI}$; w $p \mathrm{CI}$, weighted $p \mathrm{CI}$; PPC, power PC theory. Each row describes a different judgment problem.

the range of values was smaller for the weighted version of $p$ CI. To capture the lack of observed effect, it would probably be necessary to set greater differences between cell weights by increasing the weight of cell $a$ or decreasing that of cell $d$. It is not possible to determine the mean weights used by the participants in this experiment because cell values were not manipulated orthogonally, so the chosen weights are of necessity an approximation. However, the main conclusion to this part of the results is that the $p \mathrm{CI}$ rule is superior in its capacity to predict main effects of $p(\mathrm{C})$ to the $\Delta P$ rule and the power PC theory.

In addition, all three interactions between $p(\mathrm{C})$ and $\Delta P$ were significant, and none of these is predicted by either the $\Delta P$ rule or the power PC theory. Table 14 shows how these interactions break down into simple effects. In general, these too are in conformity with the predictions of the $p \mathrm{CI}$ rule, as Figure 3 shows.

Predicted values were correlated with observed mean causal judgments as in Experiment 1, and the results are presented in Table 16. This time, the predictions of all the models were highly correlated with observed means. The weighted $p$ CI rule was the most successful overall, but at $a /(a+b)=.25$ the $\Delta P$ rule was the best predictor. Differences between correlations tended to be small, however. As was explained above, despite its success on the correlation measure, the $\Delta P$ rule is inferior to the $p C I$ rule because of its failure to predict the significant main effects of and interactions with $p(\mathrm{C})$.

\section{Discussion}

There was less difference between the predictive success of the various rules in this experiment than in Experiment 1 . This is not altogether surprising. Each design incorporated a wide range of values of $\Delta P$, and all models would be expected to predict mean judgments for such sets with reasonable accuracy. Moreover, the predictions of the models are all highly correlated with each other, as Table 10-12 show, and in several cases the unweighted $p C I$ rule and the $\Delta P$ rule make the same prediction. Thus, predictive success of one model could be attributed to the fact that its predictions are highly correlated with those of another model that truly represents the computational aspect of the process of judgment, rather than to its own validity. The most discriminative test naturally lies with comparisons between judgmental problems for which the predictions of the models differ. In this case, the weighted $p C I$ rule was the most successful, mainly because of its capacity to predict effects of the $p(C)$ manipulation that were not predicted by the other models.

\section{GENERAL DISCUSSION}

These two experiments have shown that the $p$ CI rule successfully predicts a large set of observed tendencies in causal judgment. In Experiment 1, the rule predicted changes in judgment for given values of $\Delta P$ as $p(C)$ and $a /(a+b)$ were manipulated. In Experiment 2, the rule predicted changes in judgment for given values of $a /(a+$ $b)$ as $p(C)$ and $\Delta P$ were manipulated. In both cases, a weighted version of $p C I$, using weights derived from a previous experiment, was a better predictor of observed

Table 13

F Ratios, Experiment 2

\begin{tabular}{clrrrr}
\hline Design & \multicolumn{1}{c}{ Effect } & \multicolumn{1}{c}{$F$} & \multicolumn{1}{c}{$d f$} & \multicolumn{1}{c}{$M S_{\mathrm{e}}$} & \multicolumn{1}{c}{$p$} \\
\hline$a /(a+b)=.75$ & $p(\mathrm{C})$ & 27.39 & 2,82 & $1,778.22$ & $<.001$ \\
& $\Delta P$ & 86.59 & 4,164 & $1,207.72$ & $<.001$ \\
& Interaction & 5.82 & 8,328 & 934.28 & $<.001$ \\
$a /(a+b)=.50$ & $p(\mathrm{C})$ & 10.56 & 2,82 & $1,344.74$ & $<.001$ \\
& $\Delta P$ & 58.21 & 4,164 & $1,210.91$ & $<.001$ \\
& Interaction & 9.02 & 8,328 & 808.34 & $<.001$ \\
$a /(a+b)=.25$ & $p(\mathrm{C})$ & 1.34 & 2,82 & $1,332.88$ & n.s. \\
& $\Delta P$ & 79.27 & 4,164 & $1,373.14$ & $<.001$ \\
& Interaction & 3.09 & 8,328 & 993.79 & $<.01$ \\
\hline
\end{tabular}


Table 14

Simple Effects Analyses of Interactions, Experiment 2

\begin{tabular}{clrrl}
\hline \multicolumn{1}{c}{ Design } & \multicolumn{1}{c}{ Effect } & \multicolumn{1}{c}{$F$} & \multicolumn{1}{c}{$p$} & \multicolumn{1}{c}{ Differences } \\
\hline$a /(a+b) .75$ & $p(\mathrm{C})=.2$ & 56.44 & $<.001$ & $\Delta P-.25<0<.25$ and $.5<.75$ \\
& $p(\mathrm{C})=.5$ & 29.28 & $<.001$ & $\Delta P-.25$ and $0<.25,<.5$, and .75 \\
& $p(\mathrm{C})=.8$ & 23.93 & $<.001$ & $\Delta P-.25<$ all other conditions; 0 and $.25<.75$ \\
& $\Delta P=+.75$ & 0.46 & n.s. & \\
& $\Delta P=+.5$ & 11.52 & $<.001$ & $p(\mathrm{C}) .2<.5$ and .8 \\
& $\Delta P=+.25$ & 11.23 & $<.001$ & $p(\mathrm{C}) .2$ and $.5<.8$ \\
& $\Delta P=0$ & 20.24 & $<.001$ & $p(\mathrm{C}) .2<.5<.8$ \\
& $\Delta P=-.25$ & 13.43 & $<.001$ & $p(\mathrm{C}) .2<.5$ and .8 \\
$a /(a+b) .50$ & $p(\mathrm{C})=.2$ & 50.20 & $<.001$ & $\Delta P-.5<-.25<0$ and $.25<.5$ \\
& $p(\mathrm{C})=.5$ & 27.21 & $<.001$ & $\Delta P-.5<$ all other conditions; $-.25<.25$ and $.5 ; 0<.5$ \\
& $p(\mathrm{C})=.8$ & 7.96 & $<.001$ & $\Delta P-.25<.25$ and $.5 ;-.5$ and $0<.5$ \\
& $\Delta P=+.5$ & 2.02 & n.s. & \\
& $\Delta P=+.25$ & 0.35 & n.s. & \\
& $\Delta P=0$ & 3.35 & $<.05$ & No differences \\
& $\Delta P=-.25$ & 7.53 & $<.001$ & $p(\mathrm{C}) .2<.5$ and .8 \\
& $\Delta P=-.5$ & 24.13 & $<.001$ & $p(\mathrm{C}) .2<.5<.8$ \\
$a /(a+b) .25$ & $p(\mathrm{C})=.2$ & 54.84 & $<.001$ & $\Delta P-.75<-.5<-.25<0$ and .25 \\
& $p(\mathrm{C})=.5$ & 41.26 & $<.001$ & $\Delta P-.75$ and $-.5<$ all other conditions; $-.25<.25$ \\
& $p(\mathrm{C})=.8$ & 13.84 & $<.001$ & $\Delta P-.75<$ all other conditions; $-.5<0$ and $.25 ;-.25<.25$ \\
& $\Delta P=+.25$ & 1.65 & n.s. & \\
$\Delta P=0$ & 1.54 & n.s. & \\
$\Delta P=-.25$ & 2.17 & n.s. & \\
$\Delta P=-.5$ & 2.53 & n.s. & \\
$\Delta P=-.75$ & 6.60 & $<.01$ & $p(\mathrm{C}) .2<.5$ and .8 \\
& $\Delta P=1$ & & \\
& & & &
\end{tabular}

tendencies than the unweighted version of the rule. The $\Delta P$ rule and the power $P C$ theory failed to predict many of the observed tendencies.

The resemblance between the observed and predicted means is not absolutely precise. For example, in Experiment 1 , when $\Delta P=0$ the observed means for $p(\mathrm{C})=$ .2 did not exhibit the predicted differentiation (compare panel $\mathrm{F}$ of Figure 1 with panel $\mathrm{C}$ of Figure 2). In Experiment 2 , when $a /(a+b)=.25$ the observed means conformed to the predicted interaction but showed less differentiation than predicted (compare panel $\mathrm{F}$ of Figure 3 with panel $\mathrm{C}$ of Figure 4). A certain amount of chance fluctuation is to be expected. However, it does appear that there is generally less-than-predicted differentiation between means when $p(\mathrm{C})=.2$. When $p(\mathrm{C})=$ .2 , there are only eight instances per problem in which the cause is present. People tend to give more weight to cause-present than to cause-absent information (J. R. Anderson \& Sheu, 1995; Kao \& Wasserman, 1993; Levin et al., 1993; Mandel \& Lehman, 1998; Wasserman et al., 1990). With so few instances of their favored kind of information, the participants may have been less confident about their judgments. This could have induced them to make more conservative judgments, and reduced differentiation may have resulted from this.

This research has not directly tested the predictions of the Rescorla-Wagner model of associative learning in its application to human causal judgment (Allan, 1993; Rescorla \& Wagner, 1972; Shanks, 1995). It is uncertain whether the model generates predictions for these experiments because of the stimulus presentation procedure employed. The model requires an instance-by-instance presentation procedure so that there is an opportunity for an associative bond between cue (cause) and outcome (effect) to develop over a sequence of instances. It does not directly predict judgmental tendencies when contingency information is presented in a summarized form or otherwise, by being described rather than directly experienced by the participant (Shanks, 1991). In the procedure used here, the participants encounter a list of instances for each problem. This could be described as an instanceby-instance presentation procedure, inasmuch as the participants could scan the list one instance at a time from top to bottom. However, the procedure does not require them to do that: They could instead scan back and forth at will or even compile summary representations of the information.

However, the Rescorla-Wagner model was tested against the $p C I$ rule in a study by White (2003c) using an instance-by-instance presentation procedure. The model includes two learning rate parameters, one for instances

Table 15

Mean Unweighted and Weighted $p C I$ Values Compared With Observed Means for Main Effects of $\boldsymbol{P}(\mathrm{C})$, Experiment 2

\begin{tabular}{ccccr}
\hline \multirow{2}{*}{$a /(a+b)$} & \multicolumn{3}{c}{ Means } \\
\cline { 2 - 5 } .75 & $p(\mathrm{C})$ & Observed & $p$ CI & w $p$ CI \\
\hline \multirow{2}{*}{.50} & .2 & +11.94 & +.10 & +.02 \\
& .8 & +29.80 & +.25 & +.28 \\
& .2 & +42.23 & +.40 & +.49 \\
.25 & .5 & -3.79 & 0.00 & -.13 \\
& .8 & +1.94 & 0.00 & -.02 \\
& .2 & +12.43 & 0.00 & +.09 \\
& .5 & -29.92 & -.10 & -.29 \\
& .8 & -24.25 & -.25 & -.35 \\
& .8 & -25.91 & -.40 & -.37 \\
\hline
\end{tabular}



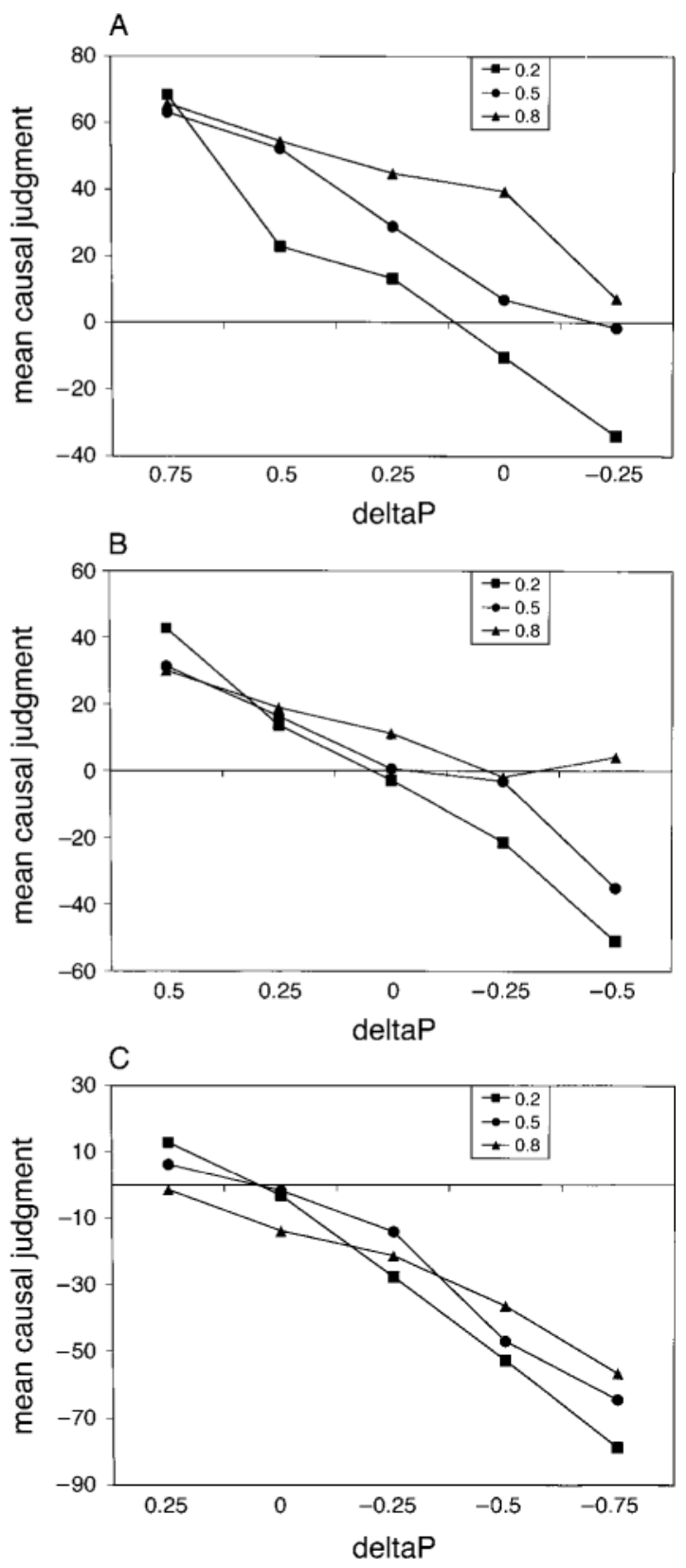

Figure 4. Mean causal judgments, Experiment 2. Panels A, B, and $C$ show means for $a /(a+b)=.75, .5$, and .25 , respectively. The key to each panel identifies values of $p(C)$.

in which the effect occurs and one for instances in which the effect does not occur. If these two learning rates are equal, then asymptotic values generated by the RescorlaWagner model match $\Delta P$ values (Chapman \& Robbins, 1990). If the two rates are unequal, then this is no longer so. However, simulations conducted by White (2003c) with various different values for the two learning rates demonstrated that no combination of values predicted the significant effects of $p$ CI manipulations observed in that study.

White $(2003 \mathrm{c})$ found a somewhat better fit between preasymptotic output of the Rescorla-Wagner model and the experimental results. However, there were several problems with this. A good fit occurred only at the very earliest stages of acquisition, and it was found to occur because the output of the model was perfectly correlated with cell $a$ frequency, which itself was moderately correlated with $p$ CI values, as would be expected. For the Rescorla-Wagner model to provide a viable account of the results, it would be necessary to assume that causal judgment was not merely preasymptotic, but that it occurred at the very earliest stages of acquisition, after the 24 instances used in each problem of that research. This is very unlikely because, as numerous studies have found, causal judgment tends to reach asymptote after a small number of trials (approximately 12; J. R. Anderson and Sheu, 1995; Baker et al., 1989; Catena, Maldonado, \& Cándido, 1998; Lober \& Shanks, 2000; Shanks, 1985). White $(2003 \mathrm{c})$ therefore concluded that the RescorlaWagner model did not predict the results of those tests of the $p \mathrm{CI}$ rule.

The $p$ CI rule is the rule of causal judgment in the EE model. This approach to causal judgment models humans as naive hypothesis testers. Usually, in studies of this sort the hypothesis is simply that the causal candidate causes the effect and people transform instances of contingency information into evidence with which to evaluate the hypothesis. The $p$ CI rule shown in Equation 2 is the specific form of the rule for the case in which there is a single causal candidate. The general principle of judging from the proportion of confirming instances yields several other versions of the $p$ CI rule for other cases. These include interactions between two causal candidates (White, 2002b), causal interpretations specifying both a cause and an enabler (a condition that makes it possible for a cause to produce its effect; White, 2000b), and causal loci in the Kelleyan causal attribution experimental paradigm (White, 2002a). In all cases, evidence supportive of the $p C I$ rule has been found.

This theoretical approach to causal judgment differs from the two approaches that have dominated recent research on causal judgment: the associative learning approach, exemplified by the Rescorla-Wagner model in its application to human causal judgment (Allan, 1993; Rescorla \& Wagner, 1972; Shanks, 1995) and the inductive rule approach, exemplified by the probabilistic contrast model (Cheng \& Novick, 1992) and the power PC theory (Cheng, 1997). Under this approach, causal judgment resembles other sophisticated judgmental activities such as jury decision making and impression formation (N. H. Anderson, 1981; Carlson \& Dulany, 1988; Catena et al., 1998; Hogarth \& Einhorn, 1992; Pennington \& Hastie, 1992). The activity of judgment proceeds with the aid of acquired notions of evidential value. White (2000a) showed that individual judgmental tendencies were well predicted by rules reported by individual participants, which in many cases deviated significantly from the normative notions of evidential value implicit in the $\Delta P$ rule. These results support the contention that causal judgment depends on acquired notions of the evidential value of different kinds of contingency informa- 
Table 16

Correlations Between Predicted and Observed Mean Causal Judgments, Experiment 2

\begin{tabular}{ccccc}
\hline & \multicolumn{4}{c}{ Model } \\
\cline { 2 - 5 }$a /(a+b)$ & $p$ CI & w $p$ CI & $\Delta P$ & PPC \\
\hline .75 & +.95 & +.95 & +.86 & +.83 \\
.50 & +.94 & +.96 & +.87 & +.86 \\
.25 & +.89 & +.89 & +.96 & +.90 \\
All & +.92 & +.96 & +.91 & +.91 \\
\hline
\end{tabular}

tion. As successive instances are presented, judgments are revised following evaluation of the evidence presented in accordance with the individual's notions of evidential value.

Catena et al. (1998) proposed a rule for asymptotic judgment that resembles the $p$ CI rule. This is shown in Equation 5.

$$
\text { New Evidence }=\frac{\left(\mathrm{w}_{1} \times a+\mathrm{w}_{2} \times b+\mathrm{w}_{3} \times c+\mathrm{w}_{4} \times d\right)}{(a+b+c+d)}
$$

This rule differs from the $p$ CI rule only in not distinguishing weights and values: Weights in Equation 5 can take positive or negative values. However, the distinguishing principle of the EE model is the partition of instances into confirmatory and disconfirmatory sets, and this feature generates predictions that distinguish the EE model from the Catena et al. model. In particular, the EE model predicts that when one kind of confirming instance is absent, greater weight is given to the other, and that the same is true for disconfirming instances. This prediction was supported by the results of two experiments by White (2003a). Those results therefore favor the EE model over the Catena et al. model. This notwithstanding, Catena et al. proposed a model for the acquisition of causal judgments over a series of trials, and the EE model has not yet been extended to the issue of acquisition. It may be that some integration of the EE model (for asymptotic judgments) and the Catena et al. model (for acquisition) would constitute a viable and comprehensive account of causal judgment, but this is a matter for the future.

The EE model therefore has a good deal of supporting evidence. It remains to be seen how well it can account for other phenomena of causal judgment, such as cue interaction effects (Baker, Mercier, Vallée-Tourangeau, Frank, \& Pan, 1993; Chapman \& Robbins, 1990; Dickinson, Shanks, \& Evenden, 1984; Price \& Yates, 1993), which are well explained by associative learning models (Shanks, 1995) and by the power PC theory (Cheng, 1997). However, any viable model of causal judgment must be capable of predicting how causal judgments are made about a single causal candidate, and in particular it must be capable of predicting the present results. The $p C I$ rule does this. It does not appear that any other current model has this capacity.

\section{REFERENCES}

Allan, L. G. (1993). Human contingency judgments: Rule based or associative? Psychological Bulletin, 114, 435-448.

Anderson, J. R., \& SHeU, C.-F. (1995). Causal inferences as perceptual judgments. Memory \& Cognition, 23, 510-524.

ANDERSON, N. H. (1981). Foundations of information integration theory. New York: Academic Press.

Baker, A. G., Berbrier, M., \& Vallée-Tourangeau, F. (1989). Judgments of a $2 \times 2$ contingency table: Sequential processing and the learning curve. Quarterly Journal of Experimental Psychology, 41B, 65-97.

Baker, A. G., Mercier, P., Vallée-Tourangeau, F., Frank, R., \& PAN, M. (1993). Selective associations and causality judgments: The presence of a strong causal factor may reduce judgments of a weaker one. Journal of Experimental Psychology: Learning, Memory, \& Cognition, 19, 414-432.

BUSEMEYER, J. R. (1991). Intuitive statistical estimation. In N. H. Anderson (Ed.), Contributions to information integration theory (Vol. 1, pp. 187-215). Hillsdale, NJ: Erlbaum.

Carlson, R. A., \& Dulany, D. E. (1988). Diagnostic reasoning with circumstantial evidence. Cognitive Psychology, 20, 463-492.

Catena, A., Maldonado, A., \& Cándido, A. (1998). The effect of the frequency of judgment and the type of trials on covariation learning. Journal of Experimental Psychology: Human Perception \& Performance, 24, 481-495.

Chapman, G. B., \& Robbins, S. J. (1990). Cue interaction in human contingency judgment. Memory \& Cognition, 18, 537-545.

Cheng, P. W. (1997). From covariation to causation: A causal power theory. Psychological Review, 104, 367-405.

Cheng, P. W., \& Novick, L. R. (1990). A probabilistic contrast model of causal induction. Journal of Personality \& Social Psychology, 58, 545-567.

CHENG, P. W., \& Novick, L. R. (1992). Covariation in natural causal induction. Psychological Review, 99, 365-382.

Dickinson, A., ShanKs, D. R., \& EvendEN, J. L. (1984). Judgement of act-outcome contingency: The role of selective attribution. Quarterly Journal of Experimental Psychology, 36A, 29-50.

HogarTh, R. M., \& EINHORN, H. J. (1992). Order effects in belief updating: The belief-adjustment model. Cognitive Psychology, 24, 155.

JENKINS, H. M., \& WARD, W. C. (1965). Judgment of contingency between responses and outcomes. Psychological Monographs: General \& Applied, 79 (1, Whole No. 594).

KaO, S.-F., \& Wasserman, E. A. (1993). Assessment of an information integration account of contingency judgment with examination of subjective cell importance and method of information presentation. Journal of Experimental Psychology: Learning, Memory, \& Cognition, 19, 1363-1386.

KePPEL, G., SAUfley, W. H., \& ToKunaga, H. (1992). Introduction to design and analysis: A student's handbook. New York: Freeman.

Levin, I. P., Wasserman, E. A., \& KaO, S. F. (1993). Multiple methods for examining biased information use in contingency judgments. Organizational Behavior \& Human Decision Processes, 55, 228-250.

LOBER, K., \& SHANKS, D. R. (2000). Is causal induction based on causal power? Critique of Cheng (1997). Psychological Review, 107, 195212.

MANDEL, D. R., \& LEhMAN, D. R. (1998). Integration of contingency information in judgments of cause, covariation, and probability. Journal of Experimental Psychology: General, 127, 269-285.

MCKenZIE, C. R. M. (1994). The accuracy of intuitive judgment strategies: Covariation assessment and Bayesian inference. Cognitive Psychology, 26, 209-239.

PenNington, N., \& Hastie, R. (1992). Explaining the evidence: Tests of story model for juror decision making. Journal of Personality \& Social Psychology, 62, 189-206.

Price, P. C., \& YATES, J. F. (1993). Judgmental overshadowing: Further evidence of cue interaction in contingency judgment. Memory \& Cognition, 21, 561-572.

ResCORLA, R. A., \& WAGNER, A. R. (1972). A theory of Pavlovian con- 
ditioning: Variations on the effectiveness of reinforcement and nonreinforcement. In A. H. Black \& W. F. Prokasy (Eds.), Classical conditioning II: Current research and theory (pp. 64-99). New York: Appleton-Century-Crofts.

SCHUSTACK, M. W., \& STERnberg, R. J. (1981). Evaluation of evidence in causal inference. Journal of Experimental Psychology: General, 110, 101-120.

SHANKS, D. R. (1985). Continuous monitoring of human contingency judgment across trials. Memory \& Cognition, 13, 158-167.

SHANKS, D. R. (1987). Acquisition functions in contingency judgment. Learning \& Motivation, 18, 147-166.

SHANKS, D. R. (1991). On similarities between causal judgments in experienced and described situations. Psychological Science, 2, 341350.

SHANKs, D. R. (1993). Human instrumental learning: A critical review of data and theory. British Journal of Psychology, 84, 319-354.

SHANKs, D. R. (1995). The psychology of associative learning. Cambridge: Cambridge University Press.

ShanKs, D. R., \& Dickinson, A. (1987). Associative accounts of causality judgment. In G. H. Bower (Ed.), The psychology of learning and motivation (Vol. 21, pp. 229-261). New York: Academic Press.

WARD, W. C., \& JENKINS, H. M. (1965). The display of information and the judgment of contingency. Canadian Journal of Psychology, 19, 231-241.

Wasserman, E. A., Chatlosh, D. L., \& Neunaber, D. J. (1983). Perception of causal relations in humans: Factors affecting judgments of response-outcome contingencies under free-operant procedures. Learning \& Motivation, 14, 406-432.

WASSERMAN, E. A., Dorner, W. W., \& KAO, S.-F. (1990). Contributions of specific cell information to judgments of interevent contingency. Journal of Experimental Psychology: Learning, Memory, \& Cognition, 16, 509-521.

Wasserman, E. A., Elek, S. M., Chatlosh, D. L., \& Baker, A. G. (1993). Rating causal relations: The role of probability in judgments of response-outcome contingency. Journal of Experimental Psychology: Learning, Memory, \& Cognition, 19, 174-188.

Wasserman, E. A., KaO, S.-F., Van HaMme, L. J., Katagiri, M., \& Young, M. E. (1996). Causation and association. In D. R. Shanks, K. J. Holyoak, \& D. L. Medin (Eds.), The psychology of learning and motivation: Vol. 34. Causal learning (pp. 207-264). San Diego: Academic Press.

White, P. A. (2000a). Causal judgment from contingency information:
Relation between subjective reports and individual tendencies in judgment. Memory \& Cognition, 28, 415-426.

White, P. A. (2000b). Causal judgment from contingency information: The interpretation of factors common to all instances. Journal of Experimental Psychology: Learning, Memory, \& Cognition, 26, 10831102.

White, P. A (2002a). Causal attribution from covariation information: The evidential evaluation model. European Journal of Social Psychology, 32, 667-684.

White, P. A. (2002b). Causal judgement from contingency information: Judging interactions between two causal candidates. Quarterly Journal of Experimental Psychology, 55A, 819-838.

WHITE, P. A. (2002c). Perceiving a strong causal relation in a weak contingency: Further investigation of the evidential evaluation model of causal judgement. Quarterly Journal of Experimental Psychology, 55A, 97-114.

W HITE, P. A. (2003a). Causal judgment as the evaluation of evidence: The use of confirmatory and disconfirmatory information. Quarterly Journal of Experimental Psychology, 56A, 491-513.

WHITE, P. A. (2003b). Effects of wording and stimulus format on the use of contingency information in causal judgment. Memory \& Cognition, 31, 231-242.

WHITE, P. A. (2003c). Making causal judgments from contingency information: The $p$ CI rule. Journal of Experimental Psychology: Learning, Memory, \& Cognition, 29, 710-727.

\section{NOTE}

1. This is necessary because under the EE model causal judgment is an estimation task (Catena, Maldonado, \& Cándido, 1998). In an estimation task, the impact of a piece of information on judgment depends on its relation to the previous judgment. Suppose the previous judgment was +.4 . If the next instance has a value of +.7 and a weight of .3 , then judgment will increase. If the instance has a value of +.7 and a weight of .1, then judgment will increase by a smaller amount. But if the instance has a value of +.2 and a weight of .3 , then judgment will decrease, even though the value of the instance is confirmatory. This is why weight and value have to be represented separately in the $p \mathrm{CI}$ rule.

(Manuscript received August 12, 2002; revision accepted for publication March 21, 2003.) 